\title{
Rester connecté à tout prix? Vulnérabilité des infrastructures critiques et gestion de crise
}

\section{Valérie November, Lausanne}

\section{Introduction}

Les infrastructures critiques font l'objet aujourd'hui d'une attention accrue et l'actualité récente - le tremblement de terre au Japon du 11 mars 2011 et ses effets collatéraux - ne font que la renforcer. Une attention politique tout d'abord, au niveau suisse: un programme de protection des infrastructures critiques a été lancé il y a deux ans par l'Office fédéral de la protection de la population (OFPP), suite à l'adoption par le Conseil fédéral le 5 juin 2009 de la stratégie générale pour la protection des infrastructures critiques. Depuis 2010, le Conseil fédéral fait l'objet d'interpellations régulières de la part de parlementaires inquiets de la qualité et de la maintenance des infrastructures. La même préoccupation est présente sur la scène européenne (KRöGER 2008) et états-unienne (AMIN 2010). Une attention scientifique accrue également: la notion d'«infrastructure critique» est récente et peine à être exactement définie (Galland 2010; Rostum, NovemBER \& VATN 2008), même s'il est admis qu'elle s'applique aux fonctions vitales de la société, celles dont la défaillance ou la perturbation créeraient des goulets d'étranglements durables dans l'approvisionnement, des dysfonctionnements importants de la sécurité publique ou d'autres conséquences dramatiques. Leurs particularités proviennent du fait que la plupart d'entre elles appartiennent ou sont exploitées par le secteur privé, ce qui ajoute un élément de complexité et oblige les gouvernements et le secteur privé à collaborer pour développer une stratégie commune de protection (GALLAND 2010).

Cependant, que sait-on exactement du bon fonctionnement des infrastructures critiques? Cette question a été tout d'abord prise en charge par la littérature scientifique en termes d'analyse de réseaux et des «large technological systems» (CouTARD 1999; OFFneR 1996) et en termes de vulnérabilité inhérente à ces systèmes, ou causée par eux (ADGER 2006; BARA 2010; Cutter 1996; Gnansounou 2008; Rosas-Casals, VALVERDE \& SOLÉ 2007).

Si l'approche systémique a été longtemps dominante et si elle a permis à des réflexions d'émerger en termes d'interdépendance et d'interconnexion des systèmes (voir Kröger 2008; Rinaldi, Peerenboom \& Kelly 2001; Zimmerman 2001), la démarche des Science and
Technology Studies (STS) a elle aussi proposé nombre de perspectives pour comprendre ces technologies et leur rôle moteur pour nos sociétés. Les recherches dans ce domaine ont mis l'accent sur des phénomènes rarement étudiés par la perspective systémique, notamment le travail réalisé en arrière-plan par des acteurs dont la performance est considérée d'autant meilleure que le résultat de leur travail demeure invisible (Bowker \& Star 1999; Millerand \& Bowker 2008). Cela a ainsi donné lieu à des travaux axés sur le travail de maintenance et de soutien technique qui ne devient apparent qu'en cas de problèmes (voir Graham \& THRIFT 2007; SHAPIN 1989).

En géographie, la perspective systémique a eu beaucoup de succès, notamment pour éclairer les aspects économiques et politiques de la question (BRUNN, Cutter \& Harrington 2004; Pecqueur \& PeyracheGadeAu 2010; Truffer 2008). Quant à la perspective STS, elle aurait été négligée au sein des recherches sur les technologies dans les études urbaines et les recherches sur les infrastructures en géographie (CouTARD \& GuY 2007; GRAHAM 2010; Hommels 2005), à l'exception notable des travaux de HinchCliffe (1996). Aujourd'hui, la rencontre a bel et bien eu lieu, si l'on considère les récentes publications intégrant géographie et STS comme l'une des clés de la compréhension du fonctionnement des infrastructures critiques (Furlong 2010; Truffer 2008). Ainsi, si les usages géographiques de l'Actor-Network Theory sont courants - à l'instar notamment de ThrifT (1996), WHATMORE (2002), November (2002, 2011), HinchClifFe et al. (2007) -, ils demeurent récents dans l'étude des infrastructures critiques. Cet article s'inscrit dans cette lignée de travaux, en y intégrant la notion de risque.

\section{Le risque comme mise en péril d'un assemblage}

Selon la définition désormais classique d'AKRICH (1987), les objets techniques définissent dans leur configuration une certaine partition du monde physique et social, attribuent des rôles à certains types d'acteurs humains et non-humains, en excluent d'autres et autorisent certains modes de relation entre ces différents acteurs. Les objets techniques sont composites et hétérogènes:

«ils renvoient toujours à une fin, une utilisation pour laquelle ils sont conçus, en même temps qu'ils ne sont qu'un terme intermédiaire sur une longue chaîne qui associe hommes, produits, outils, machines, monnaies» (AKRICH 1987:49). 
Le cadre théorique choisi s'appuie d'une part sur cette approche des techniques et la prolonge à l'aide de la notion de risque, en se concentrant sur les processus de transformation par lesquels passent les risques et les situations qu'ils vont engendrer. Il s'agit, d'autre part, de prendre au sérieux l'appel que lançait il y a quelques années RAFFESTIN (1998) aux géographes en les exhortant à se préoccuper davantage de changement que de permanence. La notion de risque a précisément été étudiée dans cette double perspective, en la définissant comme

«un événement potentiel, qui ne s'est pas encore produit, mais dont on pressent qu'il se transformera en événement néfaste (une crise) pour les individus ou pour une collectivité dans un ou des espaces donnés» (November 2003: 370).

Ainsi, il s'agit d'examiner ce que le risque «fait faire» à la société contemporaine, comment les risques sont un des moteurs essentiels de la transformation de nos territoires et sociétés, en même temps qu'ils les questionnent sur leur capacité à faire face et à les prévenir.

A l'aide de la notion de traduction, définie comme un processus par lequel un monde social et naturel se met progressivement en forme et se stabilise (AKRICH, Callon \& Latour 2006; Callon 1986), il est reconnu aujourd'hui de penser le risque comme participant à de longues chaînes d'interactions entre les artefacts techniques, les substances naturelles, les êtres humains organisés ou désorganisés, comme l'ont montré CALLON \& Rip (1992). En effet, le risque court-circuite souvent les échelles géographiques et sociales et occasionne des allers-retours entre des logiques a priori très diverses: individuelles et collectives; subjectives et objectives; micro-géographiques et macro-géographiques. Dès lors, il est théoriquement possible d'analyser les risques comme participant à la construction d'un assemblage où questions scientifiques et techniques sont étroitement mêlées aux questions sociales et politiques.

Ainsi, les risques passent par plusieurs traductions de leur identification (qui suppose de saisir les signes annonciateurs, de mettre en place des diagnostics, des critères et des indicateurs), à leur gestion (mise en œuvre de la minimisation des risques) et à leur éventuelle manifestation (catastrophe ou résorption, gestion après crise). Ces phases s'inscrivent à la fois dans des pratiques qui concernent une multitude d'acteurs et dans une dynamique: une situation préalablement analysée comme ne présentant pas de risque peut finir par se retrouver engagée dans une crise majeure en raison de la dégradation des infrastructures, d'effets domino, de l'évolution de paramètres climatiques, ou encore d'un report de risques induit par des projets érigés ailleurs mais dont la réalisation au cours du temps produit des effets dont l'accumulation finit par être problématique. Il s'agit alors d'examiner les risques de façon temporelle et dynamique en se penchant attentivement sur les processus de traduction par lesquels ceux-ci passent.

Cette perspective permet également de se décentrer de la catastrophe comme seul horizon des possibles en accordant plus d'importance aux petits événements qui surgissent constamment dans toute gestion des risques et qui ne se transforment pas pour autant en catastrophes. C'est pourquoi l'étude de cas développée ci-dessous ne porte pas sur une grande panne électrique, comme celles de 2003 en Italie, dans l'est des Etats-Unis et du Canada, ou de 2006 en Allemagne. Non, elle porte sur un événement mineur, sans grandes conséquences, mais qui a tout de même fait prendre conscience de la grande interconnexion des réseaux, des failles et des vulnérabilités du système électrique, et du fait que, sans électricité, la plupart des activités quotidiennes s'arrêtent brusquement.

\section{Etude de cas: la panne électrique du 18 janvier 2005 en Suisse romande}

L'étude de cas sur laquelle est basé cet article a été réalisée dans le cadre d'une recherche sur la vulnérabilité des infrastructures urbaines inscrite dans le cadre de l'Action COST C19 Proactive Crisis Management of Urban Infrastructure (voir NoveMBER et al. 2008). Il s'agit d'un incident qui a affecté la région romande de la Suisse.

\subsection{Déroulement de l'événement}

Le 18 janvier 2005, à 14h32, un éclair d'une intensité dix fois supérieure à la moyenne - un superbolt - s'abat sur une ligne à haute tension de $220^{\circ} 000$ volts exploitée par Energie Ouest Suisse (EOS - aujourd'hui Alpiq). Cet éclair tombe sur la ligne à Genève et le centre de haute tension de Verbois déclenche, provoquant la coupure des lignes entre Foretaille et Romanel et celles qui alimentent Genève (fig. 1). Automatiquement, le réseau genevois se branche sur le centre de Foretaille pendant une dizaine de minutes. En raison de la surcharge sur le réseau, la tension chute et le centre de Foretaille déclenche lui aussi, provoquant un effet domino sur les autres centres de la région. Le système de protection qui permet d'isoler la coupure de courant entre deux pylônes n'a pas fonctionné à cause du champ électromagnétique dégagé par la foudre.

Par effet domino, la panne se propage à l'Arc lémanique, de Genève à Saint-Triphon. Le courant revient progressivement à partir de $15 \mathrm{~h} 30$ à Genève, puis dès 15 h45 à Lausanne, mais la plus grande partie du bassin lémanique ne retrouve une alimentation normale que vers 16 h45. Le réseau de transport à très haute tension est vite rétabli, permettant ainsi aux Services industriels 


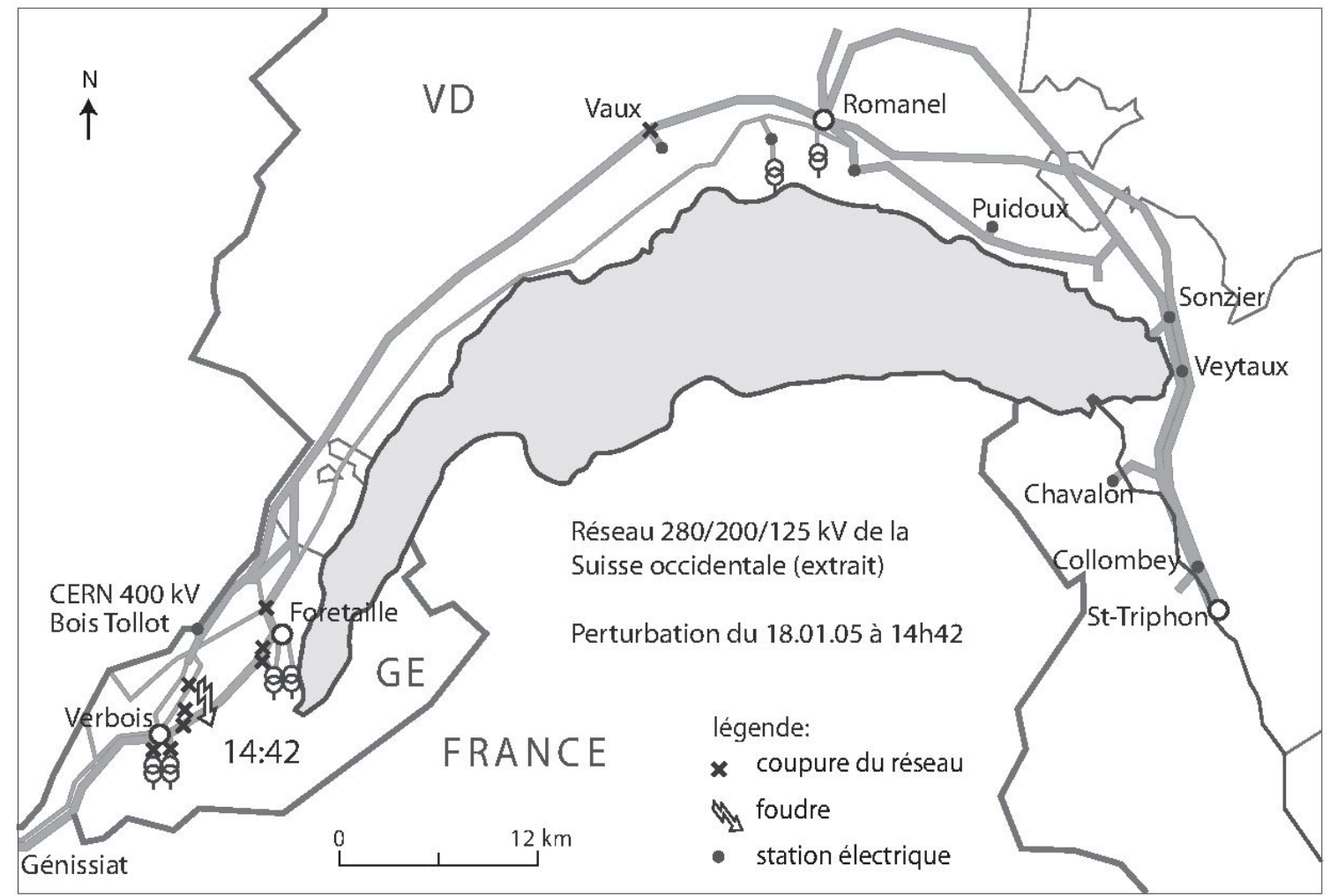

Fig. 1: Les territoires touchés par la panne électrique du 18 janvier 2005

Vom Stromausfall vom 18. Januar 2005 betroffene Gebiete

Areas hit by a power failure on January 18, 2005

Source: d'après Alpiq; cartographie: L. BAUMANN

de Genève (SIG) de réalimenter progressivement le canton de Genève. Il en est de même pour Romande Energie et les Services industriels de la Ville de Lausanne qui ont à leur tour été en mesure de réalimenter progressivement leur zone de desserte. Les acteurs estiment que le retour à la normale pour l'ensemble de l'Arc lémanique se fait à partir de 18 h00. Cette panne est la plus importante qu'ait connu la Suisse depuis 1995. Elle a touché $600^{\circ} 000$ personnes. Nombre d'activités ordinaires se sont interrompues (ascenseurs, trams et bus, feux de signalisations), provoquant embouteillages et paralysie, notamment des activités commerciales. Les entreprises et institutions dotées de générateurs ont pu continuer à assurer une partie de leurs activités, comme les Chemins de fer fédéraux, les hôpitaux ou les banques, par exemple. Au final, les entreprises de l'Arc lémanique ont subi des pertes évaluées à plusieurs dizaines de milliers de francs selon les cas.

\subsection{Méthodologie}

Les résultats sont issus d'entretiens qualitatifs, semidirectifs, auprès des acteurs impliqués dans l'étude de cas choisie, à savoir les entreprises électriques de l'Arc lémanique qui ont été affectées par la panne. A l'intérieur de celles-ci, les personnes responsables des questions de transport d'électricité, de sécurité ou de la surveillance au moment des événements ont été interviewées. Des personnes chargées de la sécurité de la population, essentiellement dans le canton de Genève, ont également été rencontrées (pour une description plus détaillée de la méthodologie et des sources analysées, voir November et al. 2008).

\section{Résultats}

Il ne s'agit pas de faire une analyse exhaustive de l'événement, mais uniquement de faire ressortir les éléments intéressants au regard de la perspective théorique esquissée ci-dessus. Nous distinguons ainsi une approche centrée sur la panne et une approche centrée sur les risques.

\subsection{Approche centrée sur la panne}

Une approche centrée sur la panne a tendance à mettre en évidence un milieu régi par des procédures 
bien délimitées, des acteurs clairement identifiés et des réseaux très hiérarchisés. Ainsi, on parlera des réseaux à très haute, moyenne et basse tension, avec chacun leurs spécificités et leurs utilisations. Cependant, dès que la panne arrive, des lignes de certains réseaux peuvent déclencher et avoir un effet de cascade. Différents lieux vont se trouver ainsi assemblés par la même panne, même si l'effet déclencheur a eu lieu à plusieurs milliers de kilomètres, comme cela a été le cas durant les black-outs nord-américain et européen de 2003. Les acteurs du secteur de l'électricité sont également clairement identifiés: on parlera de producteurs, de transporteurs, de distributeurs, de consommateurs et de traders, avec, parfois, un cumul des rôles. Des instances régulatrices interviennent également, notamment aux échelles supérieures comme l'Union pour la coordination du transport de l'électricité (UCTE) en charge des pays continentaux de l'Europe centrale et occidentale.

Toute panne met en relief un certain nombre de désaccords ou de discussions lorsqu'elle survient. Par exemple, il y a eu des malentendus dans la coordination entre les acteurs dans la gestion de la panne portant sur des questions techniques. Le réseau étant construit en cluster, la question posée était de savoir s'il fallait isoler les clusters défaillants pour protéger les autres ou, au contraire, les soutenir. D'autres éléments de discussion ont surgi lors de la reconstruction des réseaux, mettant en évidence des problèmes de circulation de l'information entre Alpiq et Romande Energie (lignes de téléphone occupées et absence de téléphone rouge). Comme le souligne une des personnes interrogées, ce problème de communication a entraîné improvisations et nécessité d'inventer d'autres solutions, mais sans pouvoir empêcher l'extension de la panne à tout le bassin lémanique.

L'état du système de transport et le manque de lignes à très haute tension constitue le troisième élément de débat présent dans ce dossier. Les personnes interrogées soulignent à quel point il est nécessaire de se montrer attentif à l'approvisionnement en énergie électrique. En effet, une interconnexion des réseaux de très haute tension ( $400^{\prime} 000$ volts) entre la Romandie et la Suisse alémanique depuis Romanel n'aurait peut-être pas permis d'éviter la panne, mais elle aurait singulièrement facilité le travail des agents d'Alpiq. Une telle connexion permettrait de mieux répartir les charges en cas de problème; le projet existe depuis plus de vingt ans, mais il n'a jamais été réalisé.

Ainsi l'approche centrée sur la panne met en évidence, d'une part, que les acteurs se connaissent tous très bien et qu'ils privilégient les compétences à l'interne de l'entreprise. D'autre part, un certain nombre de points de discussion sont présents dans ce dossier et se rapportent notamment à l'information qui n'a pas circulé de façon optimale lors de la panne. Enfin, il faut élargir le débat relativement localisé (seule la zone de l'Arc lémanique a été touchée), puisque la discussion porte sur les maillons manquants du réseau et questionne son efficacité, considérant sa position de plaque tournante en Europe.

\subsection{Approche centrée sur les risques}

L'analyse centrée sur les risques et leur traduction amène une vision supplémentaire des infrastructures critiques: elle donne à voir des approches complémentaires et l'apparition d'acteurs extérieurs au secteur de l'électricité.

Les entretiens font ressortir la présence de plusieurs approches combinées pour évaluer le risque. Ainsi, au côté d'une approche technique où le risque est une pure notion statistique, on trouve une approche contextuelle où le risque est pondéré p. ex. en fonction $\mathrm{du}$ moment, de la localisation, du temps de reprise, du type de lignes touchées, des saisons. Cette dernière approche tend à combiner les facteurs physiques avec des éléments issus de l'expérience que les acteurs ont de la situation. Les causes du black-out peuvent être alors considérées comme environnementales (nombre de black-outs ont été causés par des intempéries hors normes). Elles peuvent être aussi humaines, liées cette fois à une activité volontaire comme le terrorisme, à une cause involontaire comme une erreur sur une partie de la chaîne, ou à des questions de maintenance et d'entretien du matériel. Enfin, l'extrême interdépendance des réseaux amène les acteurs à prendre en compte des facteurs de risques associés à la vulnérabilité de leurs voisins ainsi que des autres entreprises électriques depuis une échelle cantonale jusqu'à l'échelle européenne.

Les personnes interrogées sont unanimes pour dire que la spécificité du réseau est son incroyable interconnexion aux autres réseaux. Pour pallier ce risque, l'Office fédéral de l'énergie (OFEN) a approuvé en juillet 2010 les plans déposés par Alpiq concernant la construction de la ligne à haute tension entre Chamoson et Chippis dans le canton du Valais. Cette ligne à $380 \mathrm{kV}$ comble un des chaînons manquants du réseau à très haute tension suisse. En effet, la construction de lignes à très haute tension est réclamée par les «acteurs de l'électricité». Cependant, le fait que le tracé aérien soit prôné suscite des oppositions citoyennes en Valais. Une expertise supplémentaire a été demandée à l'Ecole polytechnique fédérale de Lausanne (EPFL) pour pouvoir trancher entre l'option aérienne et souterraine.

Enfin, les entretiens ont mis en évidence des stratégies de délestage, comme le montre cette citation d'un responsable du réseau électrique: 
«Il y a des procédures de délestage, ce qui signifie qu'on sort un certain nombre de clients. En France, par exemple, des priorités sont clairement fixées, comme les casernes de police, les hôpitaux».

Ces stratégies, qui rendent certains territoires et certaines collectivités inégaux face à un approvisionnement continu en électricité, sont bel et bien une des formes (très ordinaires) de traduction du risque.

Aussi, l'approche centrée sur les risques permet de saisir plus finement comment les situations se construisent, se stabilisent parfois, et comment les choix sont opérés au niveau de leur gestion. En définitive, comme le dit un acteur interrogé:

«On pourrait dire que nous sommes des interprètes. Nous devons percevoir la situation, l'interpréter et prendre les mesures en conséquence».

\subsection{Les effets performatifs des risques et catastrophes}

Les dispositifs et les procédures internes ont été revus, renforcés et, souvent, davantage formalisés au vu de l'expérience de cette panne. C'est un processus d'apprentissage habituel dans un domaine où les acteurs sont bien conscients de la fragilité du système. Par exemple, un «téléphone rouge» a été mis en place entre les différents interlocuteurs pour éviter toute coupure des communications, un poste de «gestion des risques» et de «coordination» dans les entreprises a été créé, ainsi que des procédures de travail révisées.

La panne a également constitué un excellent argument pour concrétiser plus rapidement des projets déjà planifiés comme la création de nouveaux postes de relais. Des améliorations techniques ont également été apportées comme la désensibilisation électromagnétique pour procurer une meilleure protection aux phénomènes de foudre. La panne a permis d'accélérer des démarches qui contribuent à assurer une meilleure prévention et gestion de crise. Elle a relancé les discussions portant sur une nouvelle ligne à haute tension afin de réduire le risque d'une panne majeure en procurant davantage de possibilités d'alimentation et de gestion du réseau.

\section{Discussion}

Les résultats montrent que le risque de panne électrique fait l'objet d'une série de traductions, contribuant à reconfigurer un certain nombre d'assemblages. Cela permet une analyse en termes de temporalité et de spatialité.

Différentes échelles temporelles sont présentes dans la problématique du réseau électrique. D'abord, celui du temps quasi immédiat lorsque qu'il s'agit de réenclencher le réseau avec des procédures plus ou moins automatisées. Puis, en cas de panne, il s'agit de réagir dans un laps de temps de l'ordre de quelques minutes à plusieurs heures. Pour la maintenance, c'est une échelle de jours et de mois, alors que pour les investissements, l'échelle de travail se mesure à moyen et long terme, en années. Les plans d'investissement sont établis sur 10 à 15 ans pour le renouvellement des infrastructures, plans qui peuvent être remis en question par le contexte plus incertain provoqué par la libéralisation du marché. Il y a ainsi des tiraillements entre les objectifs de maintenance pour assurer une bonne sécurité et la nécessité d'investissements plus conséquents pour renouveler les équipements. Cette tension a tendance à s'accroître (GrahAM \& THRift 2007).

Différentes échelles géographiques se trouvent convoquées simultanément dans cette problématique. En termes spatiaux, l'ouverture des marchés, amenée par des critères essentiellement économiques, est relayée par les autorités politiques, notamment européennes, pour développer la concurrence dans ce secteur. Cela ajoute des acteurs supplémentaires liés à un niveau spatial différent, européen. Bien sûr, la connexion du réseau suisse à ceux des pays voisins est ancienne, mais dans le cadre actuel, il s'agit d'un niveau d'intégration plus poussé qui entraîne une forte augmentation des interactions entre les réseaux et les acteurs régionaux. Aussi une tension apparaît entre les besoins régionaux de renouvellement des installations et la nécessité d'assurer le transit régional et international. Ainsi, une personne interrogée exprime bien le processus de traduction à l'œuvre au niveau des échelles géographiques et de leur enchevêtrement:

«Cela dépend en fait beaucoup de la définition qu'on donne aux termes local ou régional. Pour moi, le local c'est le territoire genevois qui tient déjà compte du territoire français. La question est certainement comprise d'une autre manière si vous la posez à quelqu'un de chez EDF ou Alpiq».

L'important, ici, n'est pas de constater que l'échelle géographique de référence change selon les acteurs, mais de prendre la mesure de ce que cela change lorsqu'on s'occupe de risques et de la gestion du réseau. Il est impossible d'arrêter la gestion du risque au premier périmètre impliqué; il faut mettre celui-ci en relation avec les autres espaces concernés. Un raisonnement en termes de contiguïté doit être complété par une réflexion portant sur la connexité, à savoir la prise en compte des relations spatio-temporelles présentes simultanément (LÉvy 1994; November 2004).

\section{Conclusion}

La panne électrique qui a servi de base à l'analyse de cet article procure un certain nombre d'enseigne- 
ments. D'une part, les acteurs impliqués sont tous conscients des risques de panne et ils œuvrent tous dans leur pratique quotidienne à empêcher les incidents de générer un black-out. Cet équilibre permanent entre offre et demande, entre producteur-distributeur-consommateur, nécessite la mise en place d'un système de gestion et de contrôle pour faire face à la panne. Il y a simultanéité des temps et des espaces du risque dans le secteur des réseaux électriques. D'autre part, la panne survenue a suscité de nouvelles connaissances. Celles-ci ont été intégrées dans les systèmes de gestion du réseau électrique et de gestion de crise en cas de panne chez tous les acteurs concernés. Les principales modifications ont été apportées dans le domaine de l'organisation et de la communication.

Cette analyse montre l'importance de l'aspect humain dans la gestion des risques dans un domaine très technique. Elle révèle également l'importance stratégique des réseaux de collaboration et de l'articulation des connaissances entre les entreprises et les autorités. Une organisation formelle entre les acteurs privés et le secteur public, comme le plan OSIRIS à Genève ou ORCA dans le canton de Vaud par exemple, est nécessaire et doit être connectée aux échelles géographiques supérieures.

Alors que la Suisse va irrésistiblement vers une intégration des marchés et donc des réseaux électriques au niveau européen, la gestion des réseaux et des risques de panne prend une dimension supplémentaire. Le jeu des acteurs se complexifie davantage. Les modes de régulation et de décision engendrés par ces nouveaux impératifs économiques ne sont pas en adéquation avec les exigences liées à la simultanéité des temps et des espaces du risque mentionnées dans cet article. L'exemple étudié ici constitue un cas de figure «mineur», mais il pose les bases de ce que pourrait être un risque de black-out à l'échelle internationale. On peut donc raisonnablement se poser la question de la qualité des structures de communication entre les acteurs privés et institutionnels internationaux par rapport à leur capacité de gestion de crise. Rappelons que dans le cas étudié en Suisse, entre des acteurs qui, pourtant, se connaissent bien, c'est dans ce domaine que des lacunes sont apparues. Dans cette perspective, les enseignements de cette étude de cas de la panne survenue en Suisse le 18 janvier 2005 sur le bassin lémanique ne doivent pas être négligés. Cette panne pourrait n'être qu'un signe précurseur d'accidents plus graves, car, comme le rappelle LAGADEC (2008: 1 ),

«les risques sortent des enclos où l'on avait pu les circonscrire pour mieux les nommer, les étudier, les mesurer, les maîtriser. (...). Nous avons besoin d'un réexamen sérieux de la question des risques et des crises».

\section{Remerciements}

Cette recherche a été réalisée dans le cadre de l'Action COST C19 Proactive Crisis Management of Urban Infrastructure avec L. Boulianne, C. Cabasse et $\mathrm{M}$. Reinhardt. Elle a été financée par le Secrétariat d'Etat à l'Education et à la Recherche (Projet SER n ${ }^{\circ}$ C05.0131).

\section{Bibliographie}

AdGer, N. (2006): Vulnerability. - In: Global Environmental Change 16: 268-281.

Akrich, M. (1987): Comment décrire les objets techniques? - In: Techniques et Culture 9: 49-64.

Akrich, M., Callon, M. \& B. Latour (éds) (2006): Sociologie de la traduction. Textes fondateurs. - Paris: Ecole des Mines.

Amin, S.M. (2010): Securing the electricity grid. - In: The Bridge 40,1:13-20.

BARA, C. (2010): Social vulnerability to disasters. Zurich: Crisis and Risk Network (CRN), Center for Security Studies (CSS), Swiss Federal Institute of Technology Zurich.

Bowker, G.C. \& S.L. Star (1999): Sorting things out. Classification and its consequences. - Cambridge: MIT Press.

Brunn, S.D., Cutter, S. \& J.W. Harrington (éds) (2004): Geography and technology. - Dordrecht: Kluwer Academic Publishers.

Callon, M. (1986): Eléments pour une sociologie de la traduction: la domestication des coquilles SaintJacques et des marins-pêcheurs dans la baie de SaintBrieuc. - In: L'Année sociologique 36: 169-206.

Callon, M. \& A. RIP (1992): Humains, non-humains. Morale d'une coexistence. - In: THEys, J. \& B. KalaORA (éds): La Terre outragée. Les experts sont formels. Paris: Autrement: 140-156.

Coutard, O. (éd.) (1999): The governance of large technical systems. - Londres: Routledge.

Coutard, O. \& S. GuY (2007): STS and the city: Politics and practices of hope. - In: Science Technology \& Human Values 32, 6: 713-734.

CUTTER, S.L. (1996): Vulnerability to environmental hazards. - In: Progress in Human Geography 20, 4: 529-539.

FurLoNG, K. (2010): Small technologies, big change. Rethinking infrastructure through STS and geography. - In: Progress in Human Geography. DOI: 10.1177/0309132510380488. Publié en ligne: 1-23.

Galland, J.-P. (2010): Critique de la notion d'infrastructure. - In: Flux 81, 3: 6-18.

Gnansounou, E. (2008): Assessing the energy vulnerability: Case of industrialised countries. - In: Energy Policy 36, 10: 3734-3744.

Graham, S. (éd.) (2010): Disrupted cities. When infrastructure fails. - New York: Routledge.

Graham, S. \& N. Thrift (2007): Out of order: Under- 
standing repair and maintenance. - In: Theory, Culture \& Society $24,3: 1-25$.

HinchCliffe, S. (1996): Technology, power, and space the means and ends of geographies of technology. - In: Environment and Planning D: Society and Space 14, 6: 659-682.

Hinchcliffe, S., Kearnes, M.B., Degen, M. \& S. WhatMORE (2007): Ecologies and economies of action - sustainability, calculations, and other things. - In: Environment and Planning A 39, 2:260-282.

Hommels, A. (2005): Studying obduracy in the city: Toward a productive fusion between technology studies and urban studies. - In: Science, Technology \& Human Values 30, 3: 323-351.

KRöGer, W. (2008): Critical infrastructures at risk: A need for a new conceptual approach and extended analytical tools. - In: Reliability Engineering and System Safety 93, 12:1781-1787.

LAGADEC, P. (2008): Risques et crises: questions sur nos ancrages. - Paris: Ecole polytechnique, Cahiers du Laboratoire d'Econométrie, Dépt. d'Economie, Cahier 2008-09.

LÉvY, J. (1994): L'espace légitime. Sur la dimension géographique de la fonction politique. - Paris: Presses de Sciences Po.

Millerand, F. \& G.C. Bowker (2008): Metadata, trajectoires et «énaction». - In: LAHIRE, B. \& C. RosentaL (éds): La cognition au prisme des sciences sociales. Paris: Editions des archives contemporaines: 277-303. November, V. (2002): Les territoires des risques. Le risque comme objet de réflexion géographique. Berne: Peter Lang.

November, V. (2003): L'incendie créateur de quartier ou comment le risque dynamise le territoire. - In: Cahiers de Géographie du Québec 47, 132: 367-388.

November, V. (2004): Being close to risk. From proximity to connexity. - In: International Journal of Sustainable Development 7, 3:273-286.

November, V. (2011) (à paraître): Recalcitrance of risk: A management failure? - In: Environment and Plan$\operatorname{ning} \mathrm{A}$.

November, V., Boulianne L., Cabasse, C. \& M. ReinHARD (2008): Vulnérabilité des infrastructures urbaines et gestion de crise: impacts et enseignements dans les cas de pannes électriques en Suisse. Rapport final. Lausanne: Ecole polytechnique fédérale de Lausanne, Action COST C19.

Offner, J.-M. (1996): Réseaux et «Large Technical System». Concepts complémentaires ou concurrents? - In: Flux 12, 26: 17-30.

Pecqueur, B. \& V. Peyrache-Gadeau (2010): Fondements interdisciplinaires et systémiques de l'approche territoriale. Introduction. - In: Revue d'économie régionale \& urbaine 4: 613-623.

Raffestin, C. (1998): Permanence et changements en géographie. - In: Revue européenne des sciences sociales $36,110: 45-52$.
Rinaldi, S.M., Peerenboom, J.P. \& T.K. Kelly (2001): Identifying, understanding, and analyzing critical infrastructure interdependencies. - In: IEEE Control Systems Magazine 21, 6: 11-25.

Rosas-Casals, M., Valverde, S. \& R.V. Solé (2007): Topological vulnerability of the European power grid under errors and attacks. - In: International Journal of Bifurcation and Chaos 17, 7:2465-2475.

Rostum, J., November, V. \& J. Vatn (éds) (2008): Proactive crisis management of urban infrastructure. - Bruxelles: European Science Foundation.

SHAPIN, S. (1989): The invisible technician. - In: American Scientist 77, 6: 554-563.

Thrift, N. (1996): Spatial formations. - Londres: Sage.

Truffer, B. (2008): Society, technology, and region. Contributions from the social study of technology to economic geography. - In: Environment and Planning A 40, 4: 966-985.

Whatmore, S. (2002): Hybrid geographies. - Londres: Sage.

Zimmerman, R. (2001): Social implications of infrastructure network interactions. - In: Journal of Urban Technology 8, 3: 97-119.

\section{Résumé: Rester connecté à tout prix? Vulnérabilité des infrastructures critiques et gestion de crise}

Les infrastructures critiques font l'objet d'une grande attention du fait des pannes majeures qu'elles peuvent subir ou des cibles de malveillance potentielle qu'elles peuvent constituer. Les impacts économiques et politiques sont étudiés. Des solutions techniques de plus en plus performantes sont proposées. Cependant, ces études sont très marquées par les approches systémiques. Cet article propose de partir d'un événement a priori anodin et de mettre en évidence les mondes qu'il connecte (sociopolitiques, économiques, naturels et techniques), à l'aide d'une perspective mêlant géographie humaine et sociologie des sciences et des techniques. Une telle approche permet d'obtenir une vision plus large des risques liés aux infrastructures critiques, caractérisées par leur complexité et leur haut niveau d'interconnexion.

Mots-clés: risques, infrastructures critiques, réseau électrique, STS, échelle géographique

\section{Zusammenfassung: Vernetzt bleiben um jeden Preis? Vulnerabilität von kritischen Infrastrukturen und Krisenmanagement}

Kritische Infrastrukturen stehen im Zentrum erhöhter Aufmerksamkeit, da sie bedeutsame Defekte erleiden oder ein Ziel potentieller Zerstörung darstellen können. Es werden ökonomische und politische Auswirkungen untersucht und zunehmend leistungsfähigere technische Lösungen vorgeschlagen. Diese Studien sind jedoch stark durch systemische Ansätze 
geprägt. Mithilfe einer Perspektive, welche Humangeographie und Science, Technology, and Society Studies (STS) kombiniert, möchte der vorliegende Artikel die verschiedenen (sozialpolitischen, ökonomischen, natürlichen und technischen) Welten beleuchten, welche durch ein a priori unverfängliches Ereignis miteinander in Verbindung treten. Eine solche Perspektive ermöglicht eine breitere Sichtweise der Risiken, welche mit kritischen Infrastrukturen verbunden sind und sich durch ihre Komplexität sowie ihr hohes Mass an Vernetzung charakterisieren lassen.

Schlüsselwörter: Risiken, kritische Infrastrukturen, Stromnetz, STS, geographischer Massstab

\section{Summary: Connected at any cost? The vulnerability of critical infrastructures and crisis management}

Critical infrastructures are receiving a great deal of attention, due, on the one hand, to the major malfunctions to which they are subject and their potential as targets of malicious intent on the other. On the basis of prognoses on economic and political impacts, increasingly high-performance technological solutions are being put forth. However, these studies are very much marked by their systemic approach. In this article, the author focuses on a normally insignificant event and highlights the different fields (socio-political, economic, natural and technological) affected by the event. This approach, which combines human geography and science, technology and society studies (STS) allows for a more comprehensive view of the risks connected to critical infrastructures, and ensures that the complex and interrelated nature of such systems are taken better into account.

Keywords: risks, critical infrastructures, power grid, STS, geographical scale

Prof. Dr. Valérie November, Ecole polytechnique fédérale de Lausanne (EPFL), Faculté de l'environnement naturel, architectural et construit (ENAC), Groupe d'étude de la spatialité des risques (ESpRi), BP 2133 (Bâtiment BP), Station 16, CH-1015 Lausanne, Suisse.

e-mail:valerie.november@epfl.ch

\section{Manuskripteingang/received/manuscrit reçu le:} 14.4.2011

Annahme zum Druck/accepted for publication/accepté pour publication: 24.6 .2011 